\title{
UAV-Aided Data Delivery Scheme Based on Opportunistic Virtual Intersections for Smart Transportation Networks
}

\author{
Wei Li $\mathbb{D}^{1,2}$ Xianxing Liu, ${ }^{1,2}$ Xiaoyong Ma, ${ }^{1,2}$ Xianwei Wang, ${ }^{1,2}$ and Yi Zhou $\mathbb{D}^{1,2}$ \\ ${ }^{1}$ School of Computer and Information Engineering, Henan University, Kaifeng 475004, China \\ ${ }^{2}$ International Joint Research Laboratory for Cooperative Vehicular Networks of Henan, China \\ Correspondence should be addressed to Yi Zhou; zhouyi@henu.edu.cn
}

Received 30 May 2019; Accepted 19 August 2019; Published 18 December 2019

Academic Editor: Rakesh Mishra

Copyright (C) 2019 Wei Li et al. This is an open access article distributed under the Creative Commons Attribution License, which permits unrestricted use, distribution, and reproduction in any medium, provided the original work is properly cited.

\begin{abstract}
Smart transportation networks, featured by high mobility and intermittent connectivity, are facing critical challenges in data delivery especially when the network is sparse and with less or no support from any infrastructure, such as in disaster and military environments. Unmanned aerial vehicles (UAVs), due to their flexible mobility, can be envisioned to enhance the connectivity of ground vehicular networks. In this paper, incorporating UAVs into vehicular networks, a novel data delivery scheme, named UAVassisted data delivery (UADD), is proposed for smart transportation networks. We introduce a novel concept called "opportunistic virtual intersections", at which UAVs can communicate with ground vehicles, and assist to deliver data packets. Specifically, at an opportunistic virtual intersection, UAVs may act as a relay, which can relay data packets between two vehicles as an intermediate hop, or deliver data packets in a store-carry-and-forward manner. The data forwarding rules are devised based on expected delivery delay and delivery probability of data packets. The performance evaluation demonstrates that the proposed UAV-assisted data delivery scheme can achieve a significant improvement of data delivery performance in terms of delivery delay and delivery ratio.
\end{abstract}

\section{Introduction}

Vehicular networks have been envisioned to be critical to the next generation smart transportation networks. With vehicle-to-vehicle $(\mathrm{V} 2 \mathrm{~V})$ communications and vehicle-to-infrastructure (V2I) communications [1], vehicular networks can facilitate both vehicle safety and infotainment applications, which are considered as indispensable components for building next generation ITS [2]. However, in vehicular networks, the network performance may be compromised by the high mobility and intermittent network connectivity. For example, most of the network infrastructure can be destroyed during the disaster or war, which may disable the infrastructure-based applications; moreover, in such situations, V2V links may be blocked or the link quality may be degraded due to obstacles, complex terrains, inaccessible geographical regions, and weather conditions, among others. Therefore, vehicular delay-tolerant networks (VDTNs) can be considered as a necessary solution to deal with the intermittent connectivity and high network latency, where data can tolerate a certain level of delay, and is delivered in a store-carry-and-forward manner.
In [3], several VDTN application projects are introduced, such as KioskNet, DieselNet, CarTel, and Drive-Thru, which mostly focus on providing Internet applications through storecarry-and-forward networks. In [4], a laboratorial testbed, VDTN@Lab, is set up for evaluating the routing protocols of VDTN, in which LEGO robotic cars with Bluetooth and $802.11 \mathrm{~b} / \mathrm{g}$ are used as the miniature network models. Meanwhile, a real VDTN testbed for traffic jam and warning information services is also presented with preliminary experimental results. However, to improve the efficiency of VDTN, the key issue is to design an effective data delivery scheme including the routing protocol design and message forwarding management. In [5], the routing protocols of VDTN are investigated through comparing with the ones in vehicular ad hoc networks (VANETs), in which the VDTN routing schemes are classified by approaches based on flooding (e.g., Epidemic), probability (e.g., PRoPHET) and geography (e.g., GeoSpray). Using predictable vehicle mobility, Zhao and Cao proposed vehicle-assisted data delivery (VADD) protocols based on probabilistic traffic density information [6], in which three priority selection criteria, that is, location first, direction first, 
and hybrid, are used to select the next hop path for evaluating the performance of VADD. To decrease the overhead of message transmission, $\mathrm{Li}$ et al. considered a continuous-time Markov model to optimize the message delivery probability given an energy constraint in [7], which yields an energy-efficient opportunistic forwarding policy. In our previous work [8], we also investigated delay-tolerant data delivery for smart grid applications, in which vehicle-assisted device-to-device (V-D2D) communications are leveraged to offload cellular networks in a store-carry-and-forward mode with low cost. Later in [9], we propose a joint power control and mode selection scheme to enable V-D2D communications and analyze the two representative performance metrics, that is, SINR outage probability and link/network throughput.

The literature aforementioned focus on improving the performance of data delivery in VDTN. However, vehicular mobility is restricted by traffic pattern and road layout, which implies that the performance of VDTN is difficult to be further enhanced only relying on communications and networking among vehicles. Intuitively, if the spatial domain is extended to the aerial layer, the mobility limitation may be released and further improvement of data delivery performance is possible. Unmanned aerial vehicles (UAVs), especially mini-UAVs (e.g., quadcopters), have been found in various applications such as traffic monitoring, disaster recovery, 3D map reconstruction [10], air quality real time monitoring [11], military reconnaissance. Recently, the car manufacturer Renault has designed a "testing car" KWID including an on-vehicle quadcopter [12], where the tiny drone is expected to fly in front of the car for dangers warning on the road ahead or to avoid the traffic jam. To address aforementioned issues in VDTN, UAVs can be employed to form a cooperative air-to-ground network [13] and assist vehicular networks especially with less or no support from network infrastructure, or with very poor network connectivity. UAVs can act as relays to forward the packets among vehicles, when direct multi-hop V2V links are not available (i.e., vehicular network is disconnected). Incorporating UAVs into ground networks has attracted much research attention. In [14], Ueyama et al. employed a mini-helicopter to maintain the connectivity of wireless sensor networks (WSN) for flood detection. The helicopter serves as a mobile router [15] in multi-hop transmission and also acts as a data mule for a delay-tolerant sensor network. In [16], Tortonesi et al. investigated multi-UAV coordination and communication issues for tactical edge networks, where the middleware solutions are designed for supporting cooperative UAV networks. Considering the connectivity enhancement using a UAV, Han et al. investigated on the performance improvement of the connectivity of mobile ad hoc networks (MANETs) achieved by the UAV [17], which is evaluated through four types of network connectivity including global message connectivity, worst-case connectivity, network bisection connectivity, and k-connectivity. In [18], Goddemeier et al. further designed an agent-based role assignment strategy to provide self-optimized air-ground connectivity, where UAVs are assigned different roles through an agent state machine depending on current communication topology. In [19], Menouar et al. show the use case scenario for UAV-enabled ITS for the smart city, where one UAV could transmit the accident report/alarm through other UAVs via D2D multihop communications, and finally, to the relevant traffic entity.

It is envisioned that data deliver performance in sparse vehicular networks can be improved by incorporating UAVs. However, to the best of our knowledge, this research area is not well explored. In this paper, we propose a UAV-assisted data delivery (UADD) method to employ UAVs for assisting data delivery in VDTNs. Specifically, we first introduce a novel concept of opportunistic virtual intersections, where the routes of UAVs traverse roads. An opportunistic virtual intersection exists only when a UAV encounters a road, and vehicles on the road and the UAV can forward packets to each other. The forwarding rules are then derived by comparing the expected delivery delay and delivery probability of carrying by the UAV and the vehicle, in order to guarantee that the packet forwarding can achieve expected higher delivery performance. The main contribution of this paper is that we further improve the performance of data delivery in VDTNs by incorporating UAVs into vehicular networks.

The remainder of this paper is organized as follows. In Section 2, we first introduce the proposed UADD model and data delivery scheme in VDTN. The detailed data forwarding protocol design is presented and analyzed in Section 3. In Section 4, we present the simulation results to evaluate the performance of UADD. Our paper closes with conclusions and future work in Section 5.

\section{System Model}

In this section, we first introduce the overview of the UAVassisted vehicular delay-tolerant network and then present the proposed UADD model including communication model, road network, and mobility model.

2.1. UADD Overview. As depicted in Figure 1, in UADD, a sparse vehicular network is considered, which is a typical delay-tolerant network with intermittent connectivity and a certain level of tolerance to data delivery delay. The most significant issue is to find a data delivery routing within the shortest delay time. The UAV-assisted VDTN framework composes of two layers, which are aerial layer and ground layer, respectively. In the ground layer, all vehicles are equipped with communication modules (e.g., DSRC/IEEE 802.11p) and GPS which make them capable of both communicating and positioning. Besides, the preloaded digital map can provide vehicles with street-level statistical traffic information of the area, such as the vehicle density of different times of the day, speed limit of the vehicle on the road and traffic signal at intersections. In the aerial layer, UAVs set up an aerial network. In general, UAVs are assigned with tasks such as traffic monitoring and ground image information collection. Therefore, when UAVs move around to conduct the assigned tasks, they are considered to be able to help relay or carry and forward the VDTN packets.

2.2. Communication Model. There exist three kinds of communication links for this UAV-assisted VDTN model, that is, air-to-air (A2A) links, air-to-ground (A2G) links, and vehicle-to-vehicle (V2V) links. Since A2A links do not happen 


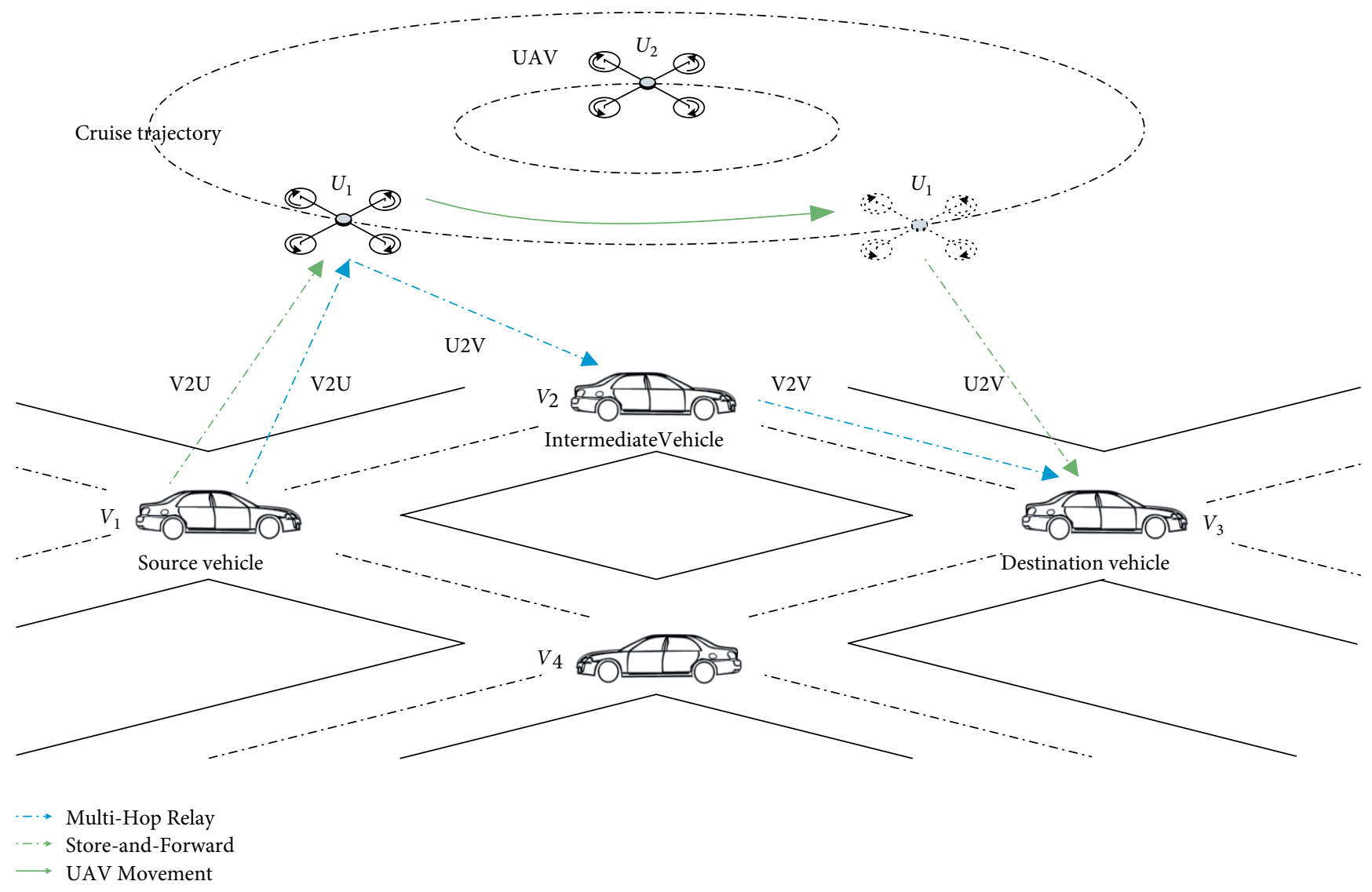

FIGURE 1: A UAV-assisted data delivery model.

frequently due to smaller number of UAVs with unconstrained movement (compared with vehicular networks where vehicles moves along roads), in the proposed UADD method, we consider both A2G and V2V links, and leave the A2A links in future works. Figure 1 shows both A2G and V2V links, where A2G links are further divided into UAV-to-vehicle (U2V) link and vehicle-to-UAV (V2U) link according to different data delivery directions. $R_{V}$ and $R_{U}$ are used to denote the communication range of vehicles and UAVs, respectively. And in this work, we set the communication range, $R_{V}$ and $R_{U}$ to

$$
100 m \leq R_{V}, R_{U} \leq 250 m .
$$

To assist the data delivery, UAVs can work in two different modes, that is, the multi-hop relay mode and store-carry-andforward mode. In VDTNs, the case of connectivity holes is one of the main reasons that degrades the data delivery performance. With the assist of UAV relaying, the connectivity hole might be addressed and a multi-hop path could be established. It can be seen from Figure 1, the source vehicle $V_{1}$ needs to forward a data packet to the destination vehicle $V_{3}$. However, a direct multi-hop path is not available. Therefore, the data delivery may rely on the intermediate vehicle $V_{2}$ and the UAV $U_{1}$ through multi-hop relay delivery, for example, link $V_{1} \rightarrow U_{1} \rightarrow V_{2} \rightarrow V_{3}$. If a multi-hop path is not available even with the relay of UAVs, the UAV can act as store-carry-andforward mode. The UAV will store and carry the data packet until it finds the better next-hop, and forwards the packet to the next-hop node. Figure 1 shows another link from $V_{1}$ to $V_{3}$, that is, $V_{1} \rightarrow U_{1}\left(\rightarrow U_{1}\right) \rightarrow V_{3}$, where the dash-arrow represents that UAV is moving carrying the data packet.

2.3. Road Network. We consider a sparse road network with eastbound-westbound roads intersecting with southboundnorthbound roads, and denote intersection $x$ by $I_{x}$. Denote by $r_{x y}$ the road segment between intersection $I_{x}$ and $I_{y}$, and by $l_{x y}$ and $\rho_{x y}$ the length of $r_{x y}$ and vehicle density on $r_{x y}$. The routes of UAVs intersect with roads and form opportunistic virtual intersections. When a UAV moves to an opportunistic virtual intersection, it can assist to relay or carry and forward data packets. As shown in Figure 2, consider that the source vehicle at $I_{s}$ sends a packet to the destination intersection $I_{d}$, and a $\mathrm{UAV}$ is moving along a rectangular route. Ten opportunistic virtual intersections are shown in the figure by blue circles. Through the cooperation among the UAV and vehicles, the data packet can be delivered from $I_{s}$ to $I_{d}$. Note that for the sake of simplicity, we do not consider the situation that an opportunistic virtual intersection is overlapped with an intersection.

2.4. Mobility Model. Vehicles move along road segment $r_{x y}$ at a varying speed $v_{V, x y} \in\left[0, v_{m, x y}\right]$, where $v_{m, x y}$ is the speed limit of $r_{x y}$. Similar to [6], we assume that the inter-vehicle distances on road segment $r_{x y}$ follow an exponential distribution with a 


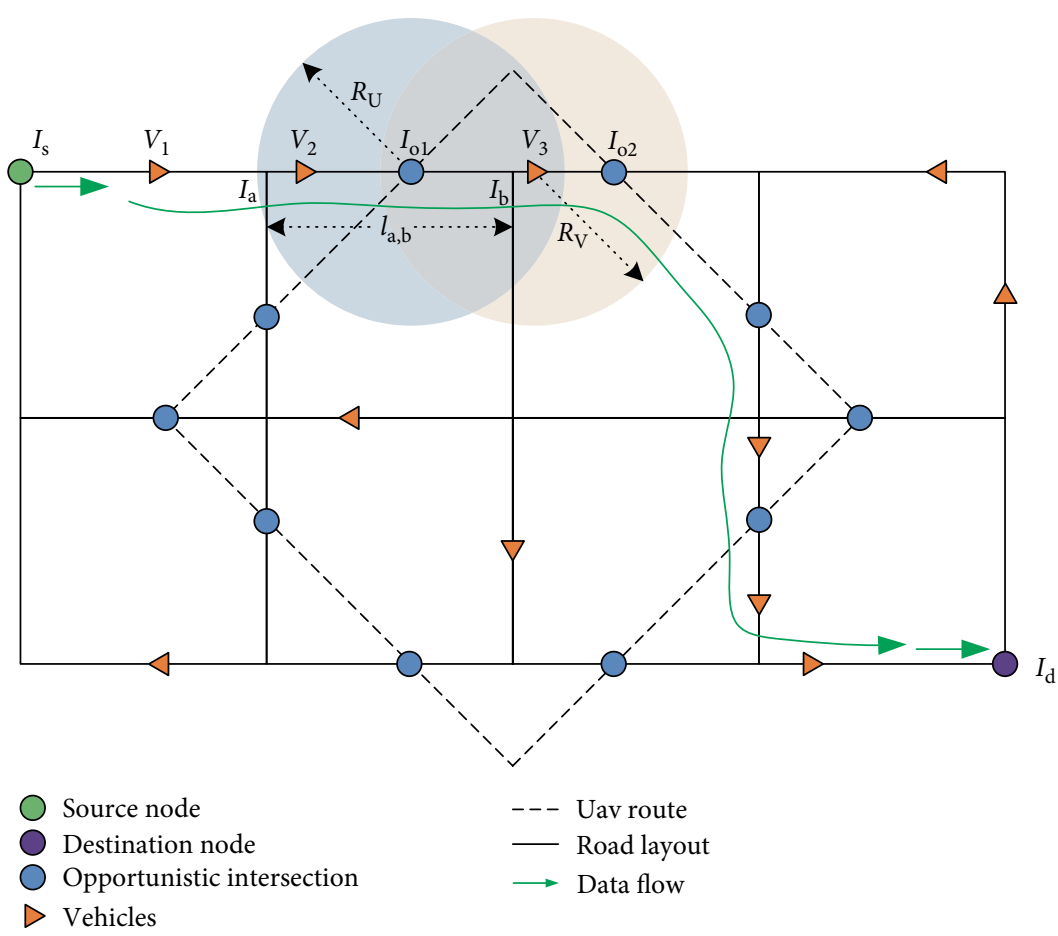

FIgURE 2: A geometrical model of UAV-assisted vehicular networks.

mean distance of $1 / \rho_{x y}$. Therefore, the expected packet delivery delay between two points $a$ and $b$ within a road segment $r_{x y}$ can be calculated by

$$
\mathscr{D}_{a b}=\left(1-e^{-R_{V} \rho_{i j}}\right) \frac{l_{a b} o}{R_{V}}+e^{-R_{V} \rho_{i j}} \frac{l_{a b}}{v_{V, i j}},
$$

where $l_{a b}$ is the distance from $a$ to $b$, and $o$ is the average onehop transmission delay between two vehicles. Equation (2) indicates that within a road segment $r_{i j}$, on a portion of $1-e^{-R_{V} \rho_{i j}}$ of the road segment, the inter-vehicle distance is smaller than $R_{V}$, where the packet is forwarded by direction transmission, while on the rest of road, the packet is carried by vehicles. UAVs moves along their predefined routes which are determined by the tasks assigned to them. Denote by $v_{U, k}$ the speed of UAV $U_{k}$. A summary of the important notations used in this paper is given in Table 1.

\section{UADD Packet Forwarding Protocol}

In this section, we describe the detailed packet forwarding protocol used in UADD method. Since the packet forwarding can happen in three different cases, that is, at intersections, at opportunistic virtual intersections, and on a straight road, we discuss the protocol according to these cases respectively.

3.1. Forwarding at Intersections and Straight Ways. Since we consider that UAVs' path does not intersect with the road intersections, the packet forwarding at intersections can be conducted in a simple way. We use the prioritized direction based forwarding method that is similar in VADD [6].
At an intersection $I_{i}$, each of the road segment $r_{i j}$ that connects $I_{i}$ to its neighboring intersections is assigned by a priority $\sigma_{i j} \in\{1,2,3,4\}$, where $\sigma=1$ is the highest priority and $\sigma=4$ is the lowest. When a vehicle $V_{a}$ carrying a packet arrives at $I_{i}$, it looks for the next-hop vehicle of the packet on the road segment with the highest priority $\sigma=1$. If there exists one vehicle $V_{b}$ on the road segment with $\sigma=1, V_{a}$ forwards the packet to $V_{b}$; otherwise, $V_{a}$ looks for vehicles on the road segment with $\sigma=2$, and so on. Priorities are assigned according to $\varphi_{i j}$, which is the angle between $r_{i j}$ and the vector from $I_{i}$ to the destination. $r_{i j}$ with a smaller value of $\varphi_{i j}$ is assigned with a higher priority since it is considered that $r_{i j}$ can lead to intersections closer to the destination with a higher probability.

As shown in Figure 3, vehicle $V_{a}$ need to forward the packet to destination $D_{o}$. After a simple comparison, the angle between the road $r_{e b}$ and the vector from $V_{a}$ to $D_{o}$, that is, $\varphi_{e b}$ has the smallest value; therefore, the road $r_{a b}$ has the highest priority with $\sigma_{e b}=1$. And in the same way, we get $\sigma_{e f}=2$, $\sigma_{e d}=3$, and $\sigma_{e h}=4$. Vehicle $V_{a}$ first checks the road $r_{e b}$ and finds no vehicle on it. Then it checks the road $r_{e f}$ and finds vehicle $V_{b}$ which is closer towards the destination $D_{o}$. Thus, vehicle $V_{a}$ forwards the packet to vehicle $V_{b}$. Up to here, the packet forwarding at the intersection $I_{e}$ has been finished. Comparing with that at intersections, packet forwarding on straight ways is much simpler, since the straight-way traffic happens only at two directions. When the packet carrier is driving on a straight way, it will keep carrying the packet until it finds a vehicle to forward ahead. Certainly, it may also occur when the packet carrier $V_{a}$ finds a vehicle $V_{b}$ in the opposite direction, from which position the estimated delay time is much smaller. As a consequence, the vehicle $V_{a}$ may decide to forward the packet to the vehicle $V_{b}$. However, such cases happen rarely, and if they happen, the computation overhead of 
TABLE 1: Notations in UADD.

\begin{tabular}{lc}
\hline Notations & Description \\
\hline$V_{x}$ & The vehicle $x$ \\
$U_{x}$ & The UAV $x$ \\
$R_{V}$ & The communication range of vehicles \\
$R_{U}$ & The communication range of UAVs \\
$I_{x}$ & The intersection $x$ \\
$r_{x y}$ & The road segment between $I_{x}$ and $I_{y}$ \\
$l_{x, y}$ & The length of the road segment $r_{x y}$ \\
$\rho_{x y}$ & The vehicle density of road $r_{x y}$ \\
$v_{V, x y}$ & The speed of the vehicle on the road $r_{x y}$ \\
$v_{U, x}$ & The speed of the UAV $x$ \\
$D_{x y}$ & The expected packet delivery delay between points \\
& $x$ and $y$ within a road segment \\
$p_{p o}$ & The packed delivery probability to the optimal next \\
& intersection $D_{o}$
\end{tabular}

the delay time will become higher. So we leave these optimizations in the future work due to space limit.

3.2. Forwarding at Opportunistic Virtual Intersections. Packet forwarding at opportunistic virtual intersections, that is, from ground vehicles to UAVs, or from UAVs to ground vehicles, is the main focus of this paper. To better depict the forwarding rule, we first introduce the concept of "optimal next-intersection" of a packet to be forwarded. As shown in Figure 4, assume a packet is on road segment $r_{i j}$, and according to the location of the destination, there are six possibilities of the optimal next-intersection of the packet. At intersection $I_{i}$, two lines divide the area to the west of $I_{i}$ into three sectors with angle $\pi / 4$ and $3 \pi / 4$, respectively. If the destination is in sectors $S_{a}, S_{b}$, and $S_{c}$, then the optimal next-intersection of the packet $D_{o}$ is $I_{a}, I_{b}$, and $I_{c}$, respectively. Similarly, if the destination locates in $S_{d}, S_{e}$, and $S_{f}$, the optimal next-intersection of the packet $D_{o}$ is $I_{d}, I_{e}$, and $I_{f}$, accordingly. Using the priorities defined in the previous part, it can be seen that the optimal next-intersection is also the intersection between which and $I_{i}$ or $I_{j}$ the road segment has the highest priority. The definition of the optimal next-intersection indicates that if the packet cannot be forwarded directly from $I_{i}$ or $I_{j}$ to the optimal nextintersection, the expected packet delivery delay will be much higher, since the packet may be routed through a path that is likely to be much longer.

Using the concept of the optimal next-intersection, we can design the forwarding rules when UAVs are involved. First, we consider the situation that a vehicle carrying the packet encounters a UAV on the road. During the encounter, the vehicle $V_{m}$ and the UAV $U_{n}$ can first exchange some information which is useful for making forwarding decision. The information can include the direction $\theta_{U}$ and speed $v_{U}$ of the $\mathrm{UAV}$, and those of the vehicles within $R_{U}$ which is the transmission range of the UAV. We define the angle between the direction of $V_{m}$ and the vector from $V_{m}$ to $D_{o}$ as $\theta_{V D}$, and the angle between the direction of $U_{n}$ and the vector from $U_{n}$ to $D_{o}$ as $\theta_{U D}$. In the following, we discuss the forwarding rules in different cases.

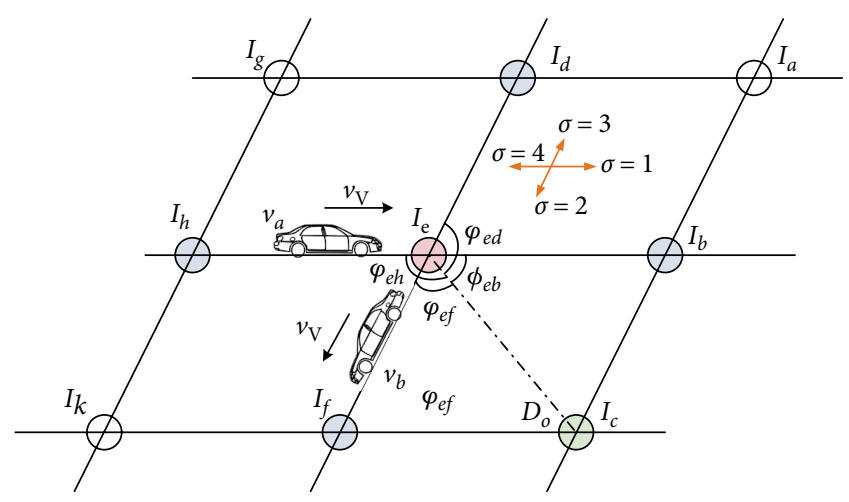

FIGURE 3: Packet forwarding at intersections.

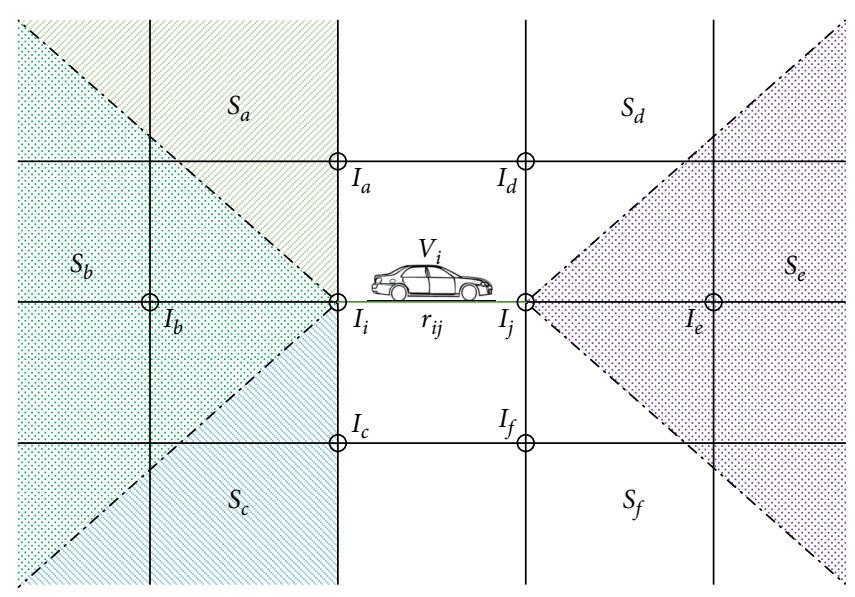

FIGURE 4: Illustration of the optimal next-intersection.

(1) $\theta_{V D}<\pi / 2$ and $\theta_{U D}>\pi / 2$ : In this case, it is roughly considered that $V_{m}$ is moving towards the destination, while $U_{n}$ is moving away from the destination. Therefore, the packet is forwarded to $U_{n}$ only if $U_{n}$ can work in relay mode and relay the packet to the vehicle $V_{m^{\prime}}$ ahead along the direction of $V_{m}$. The expected packet delivery delay that can be reduced by the relaying can be calculated by

$\Delta \mathscr{D}=\left(1-e^{-R_{V} \rho_{i j}}\right) \frac{l_{m m^{\prime}} O}{R_{V}}+e^{-R_{V} \rho_{i j}} \frac{l_{m m^{\prime}}}{v_{m}}+2 o^{\prime}$.

In Equation (3), $l_{m m^{\prime}}$ is the distance between $V_{m}$ and $V_{m^{\prime}}$, and $o$ and $o^{\prime}$ are the one-hop transmission delay between two vehicles and between a vehicle and a $\mathrm{UAV}$, respectively. $1-e^{-R_{V} \rho_{i j}}$ and $e^{-R_{V} \rho_{i j}}$ are the portions of the road on which the packet is transmitted between neighboring vehicles and on which a packet has to be carried by a moving vehicle. Therefore, the first two items of (3) are the expected delay of the packet from the current location of $V_{m}$ to the current location of $V_{m^{\prime}}$. On the other hand, if the packet is relayed by $U_{n}$, the third item $2 o^{\prime}$ is the delay.

(2) $\theta_{V D}>\pi / 2$ and $\theta_{U D}<\pi / 2$ : In this case, we consider that $V_{m}$ is moving away from the destination, while 
$U_{n}$ is moving towards the destination. As a result, the packet is forwarded from $V_{m}$ to $U_{n}$, since whether $U_{n}$ works as relay mode or as carrying mode, the packet is expected to have a shorter delay than if it is still carried by $V_{m}$. Then, if there is no vehicle $V_{m^{\prime}}$ that $U_{n}$ can relay packet to, $U_{n}$ will carry the packet. If there are candidate vehicles to relay the packet to, $U_{n}$ will relay the packet according to the rules described in the following parts.

(3) $\theta_{V D}>\pi / 2$ and $\theta_{U D}>\pi / 2$ : In this situation, both $V_{m}$ and $U_{n}$ are considered moving away from the destination. However, since $V_{m}$ is moving along the road, it is likely that $V_{m}$ can encounter a vehicle $V_{m^{\prime}}$ moving in the opposite direction, and can forward the packet to $V_{m^{\prime}}$. Therefore, in this case, the packet is not forwarded to $U_{n}$ unless $U_{n}$ can further relay the packet to $V_{m^{\prime}}$.

(4) $\theta_{V D}<\pi / 2$ and $\theta_{U D}<\pi / 2$ : In this case, both $V_{m}$ and $U_{n}$ are considered to be moving towards the destination. To decide whether forward the packet to $U_{n}$, two metrics are defined, which are the packet delivery probability to the optimal next-intersection $D_{o}$ and the expected packet delay to $D_{o}$, denoted by $p_{p o}$ and $\mathscr{D}_{p o}$, respectively. $p_{p o}$ is the probability that the packet can be delivered to $D_{o}$ through the shortest path, while $\mathscr{D}_{p o}$ is the expected delay if the packet is delivered to $D_{o}$ through the shortest path. As discussed above, if a packet cannot be delivered to $D_{o}$ through the shortest path, the total expected packet delivery delay might be very long. Therefore, we consider that larger value of $p_{p o}$ and smaller value of $\mathscr{D}_{p o}$ indicate a better packet path. Therefore, a packet is forwarded to UAV if UAV can switch to relay mode, or if the following conditions can be satisfied.

$$
\begin{aligned}
& p_{p o, U} \geq p_{p o, V} \\
& \mathscr{D}_{p o, U} \leq \mathscr{D}_{p o, V} .
\end{aligned}
$$

If the two conditions in (4) are satisfied, a store-carry-andforward packet delivery by the UAV is favored, since the packet can be delivered to the optimal next-intersection $\mathscr{D}_{o}$ with a higher probability and a lower delay. The calculation of $p_{p o, U}$, $p_{p o, V}, \mathscr{D}_{p o, U}$ and $\mathscr{D}_{p o, V}$ is given by the following example. As shown in Figure 5, a vehicle encounters a UAV at an opportunistic virtual intersection $I_{o a}$ and makes a decision whether to forward the packet to the UAV. From the figure, it can be seen that the shortest path to the optimal nextintersection $I_{b}$ is $I_{o a} \rightarrow I_{o b} \rightarrow I_{b}$ if the packet is forwarded to the UAV, and UAV acts in store-carry-and-forward mode, and is $I_{o a} \rightarrow I_{a} \rightarrow I_{b}$ if the packet is not forwarded to the UAV. Then, $\mathscr{D}_{p o, V}$ can be calculated by

$$
\begin{aligned}
\mathscr{D}_{p o, V}= & \left(1-e^{-R_{V} \rho_{a c}}\right) \frac{l_{o a, a} O}{R_{V}}+e^{-R_{V} \rho_{a c}} \frac{l_{o a, a}}{v_{V, a c}} \\
& +\left(1-e^{-R_{V} \rho_{a b}}\right) \frac{l_{a, b} O}{R_{V}}+e^{-R_{V} \rho_{a b}} \frac{l_{a, b}}{v_{V, a b}} .
\end{aligned}
$$

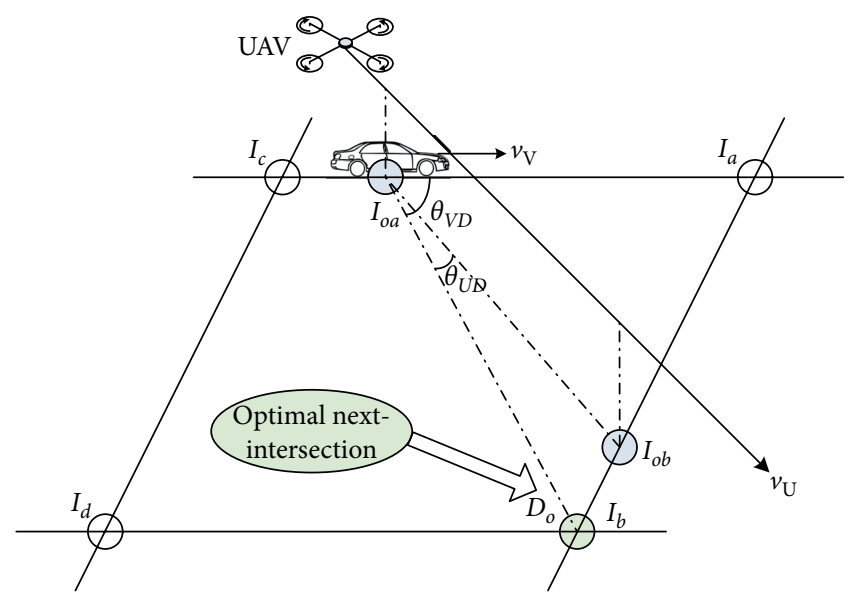

FIGURE 5: Illustration of calculation of forwarding metrics.

Similarly, $\mathscr{D}_{p o, U}$ can be calculated by

$$
\mathscr{D}_{p o, U}=\frac{l_{o a, o b}}{v_{U}}+\left(1-e^{-R_{V} \rho_{a b}}\right) \frac{l_{o b, b} O}{R_{V}}+e^{-R_{V} \rho_{a b}} \frac{l_{o b, b}}{v_{V, a b}} .
$$

Since $p_{p o, V}$ is the probability that the packet can be delivered to $I_{b}$ through the shortest path if carried by the vehicle $V_{m}$, it is straightforward that $p_{p o, V}$ can be calculated by

$$
p_{p o, V}=\operatorname{Pr}\left(V_{m} \Longrightarrow r_{a b} \cup V_{m} \rightarrow r_{a b}\right),
$$

where $V_{m} \Longrightarrow r_{a b}$ is the event that $V_{m}$ can find at least a vehicle on $r_{a b}$ to forward the packet when $V_{m}$ sojourns at $I_{a}$, and $V_{m} \rightarrow r_{a b}$ is the event that $V_{m}$ takes $r_{a b}$ to move on after passing $I_{a}$. Under the assumption of Poisson process of vehicle arrivals at the intersections, the arrival rate at $r_{a b}$, denoted by $\lambda_{a b}$, can be calculated by $\lambda_{a b}=\rho_{a b} v_{V, a b}$ according to [20]. Therefore,

$$
\operatorname{Pr}\left(V_{m} \Longrightarrow r_{a b}\right)=\operatorname{Pr}\left(N_{a b}\left(T_{a}\right) \geq 1\right)=1-e^{-\lambda_{a b} T_{a}},
$$

where $T_{a}$ is the sojourn time of $V_{m}$ at $I_{a}$, and $N_{a b}\left(T_{a}\right)$ is the number of vehicles arrived at $r_{a b}$ during $T_{a} \cdot \operatorname{Pr}\left(V_{m} \rightarrow r_{a b}\right)$ is the probability that $V_{m}$ turns to south direction at $I_{a}$, which is $p_{s}$. Assuming that $V_{m} \Longrightarrow r_{a b}$ and $V_{m} \rightarrow r_{a b}$ are independent, it can be obtained that

$$
p_{p o, V}=1-\left(1-p_{s}\right) e^{-\lambda_{a b} T_{a}}
$$

Similarly,

$$
p_{p o, U}=\operatorname{Pr}\left(U_{n} \Longrightarrow r_{b d}\right)=1-e^{-\lambda_{b d} T_{o b}},
$$

where $T_{o b}$ is the sojourn time of the UAV at $I_{o b}$. In (10), there is no item of $U_{n} \rightarrow r_{b d}$ since at the opportunistic virtual intersection, vehicles and UAVs do not change the direction of movement. Note that if the packet forwarding happens in the opposite direction, that is, from a UAV to a ground vehicle, conditions in (4) also apply. The UAV checks the conditions and keeps carrying the packet if both conditions are satisfied. Otherwise, it forwards the packet to ground vehicles. 


\section{1: Forwarding At Intersections:}

2: Assign road segments connected to the intersection with priorities $\sigma_{i j}$ according to $\varphi_{i j}$.

3: Forwarding the packet to the vehicle according to the priorities.

4: Forwarding At Opportunistic Virtual Intersections.

5: Determine the optimal next-intersection $D_{o}$.

6: Determine $\theta_{V D}$ and $\theta_{U D}$ based on $D_{o}$.

7: if $\theta_{V D}<\pi / 2$ and $\theta_{U D}>\pi / 2$ then.

8: $\quad$ Forwarded packet to $U_{n}$ only if $U_{n}$ can work in relay mode and relay the packet to the vehicle $V_{m^{\prime}}$ ahead along the direction of $V_{m}$.

9: else if $\theta_{V D}>\pi / 2$ and $\theta_{U D}<\pi / 2$ then.

10: $\quad$ Forwarded packet to $U_{n}$.

11: else if $\theta_{V D}>\pi / 2$ and $\theta_{U D}>\pi / 2$ then.

12: Forwarded packet to $U_{n}$ only if $U_{n}$ can work in relay mode and relay the packet to the vehicle $V_{m^{\prime}}$ ahead along the direction of $V_{m}$.

13: else

14: Calculate the packet delivery probability and expected packet delay of both $V_{m}$ and $U_{n}$.

15: Forward to $U_{n}$ if (4) is satisfied or $U_{n}$ can work in relay mode and relay the packet to the vehicle $V_{m^{\prime}}$ ahead along the direction of $V_{m}$.

16: end if

Algorithm 1: UADD Packet Forwarding Algorithm.

\section{Performance Evaluation}

In this section, we evaluate the proposed UADD scheme via simulation. As shown in Figure 6, the simulation scenario is a $2500 \mathrm{~m} \times 2500 \mathrm{~m}$ sparse road network. The simulation parameters are justified as follows:

(1) Vehicle Mobility: The mobility trace of 50 vehicles is generated by VANETMobisim [21]. Speed limit is set to $50 \mathrm{~km} / \mathrm{h}$, which is set according to rules in most urban areas. The vehicle mobility is controlled by Intelligent Driver Model with Lane Changes (IDMLC) model, where vehicle speed is based on movements of vehicles in neighborhood.

(2) UAV Mobility: The UAVs fly round-trip in the area to execute certain tasks, such as patrol and surveillance. Meanwhile, they can help the forwarding of packets in an opportunistic manner. In our simulation, there are $20 \mathrm{UAV}$ s, and each UAV moves round-trips between two randomly chosen locations, with speed $v_{U} \in[10,25] \mathrm{m} / \mathrm{s}$.

(3) Data Traffic: We generate the data traffic according to a constant bitrate rule, e.g., the vehicular users request the contents from the content providers. The communication ranges of vehicles and UAVs are set to $100 \mathrm{~m}$ in radius. Data packets are generated according to a constant rate $R$, and upon generation,

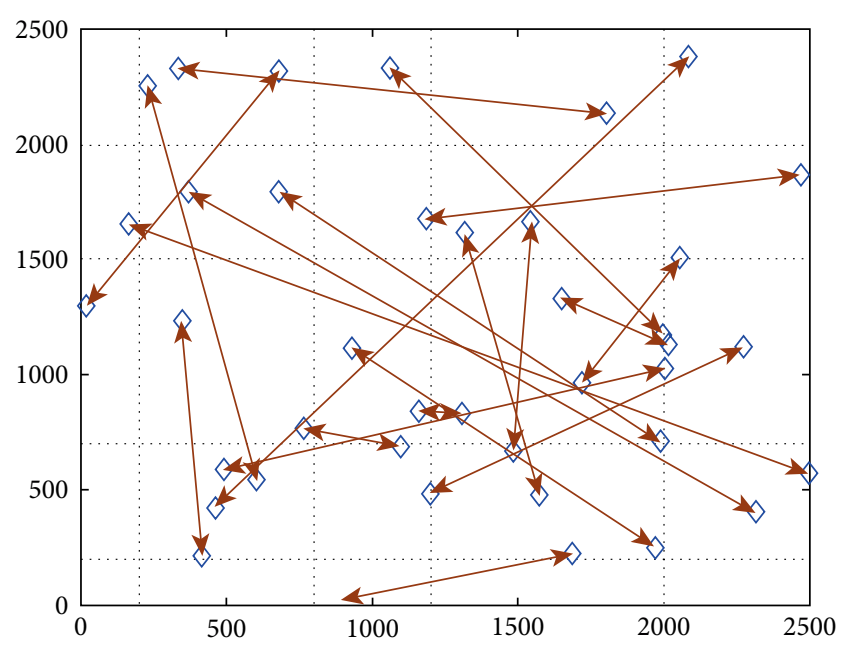

FiguRE 6: Simulation scenario.

each packet randomly chooses an intersection as the destination.

For the results, we mainly compare our results to those of VADD scheme proposed in [6] and the well known Epidemic routing protocol. In VADD, packet forwarding is based on a calculation of expected packet delivery delay, and the packet is forwarded to the vehicle that can achieve minimal expected delay. The performance comparison among VADD and other packet forwarding schemes such as DSR, and GPSR can be found in [6]. In the simulation, we set a delay tolerance $\mathscr{D}_{t}=400$ seconds for the packets such that a packet that exceeds $\mathscr{D}_{t}$ without delivery will be discarded.

4.1. The Data Delivery Ratio. The delivery ratios of data packets are shown in Figure 7 with respect to the data rate in packet per second. It can be seen that the Epidemic protocol achieves the lowest delivery ratio because a vehicle stores a copy of each packet if its buffer is not full, and thus the buffers get full easily. If the buffer of a vehicle is full, new generating packets are dropped. The reason that UADD can achieve higher delivery ratio than VADD is two-fold. First, UAVs can assist to buffer packets. Second, UADD can achieve lower packet delivery delay (discussed below), and thus buffers can be released faster than those in VADD. With the increase of data rate, more data packets are generated in the network, and therefore more packets are dropped due to full buffers, resulting in the decrease in the delivery ratios of UADD, VADD, and Epidemic.

4.2. The Average Package Delivery Delay. The average packet delivery delay with respect to the data rate is shown in Figure 8. Generally, Epidemic has the worst performance in packet delivery delay among the three, in spite that it is expected to achieve the best packet delivery delay performance due to many copies of a packet exist in the network. This is because due to the full buffer, it is less likely that packets can be forwarded to other vehicles, and thus the chance that a packet can arrive at the destination becomes small. An increase in packet delivery 


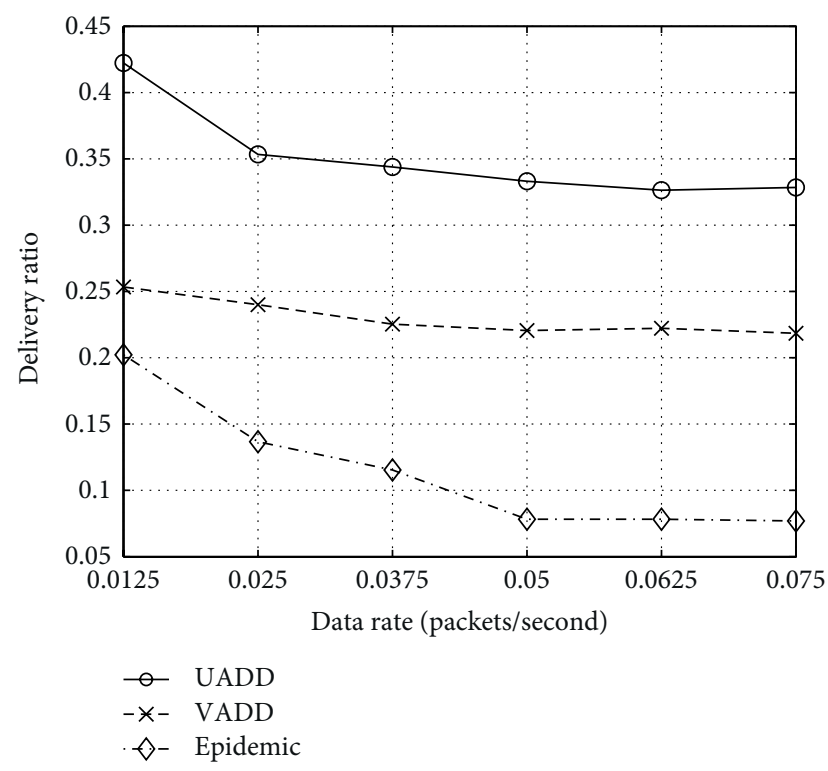

FIgURE 7: Delivery ratio. Buffers of vehicles are set to 50 packets.

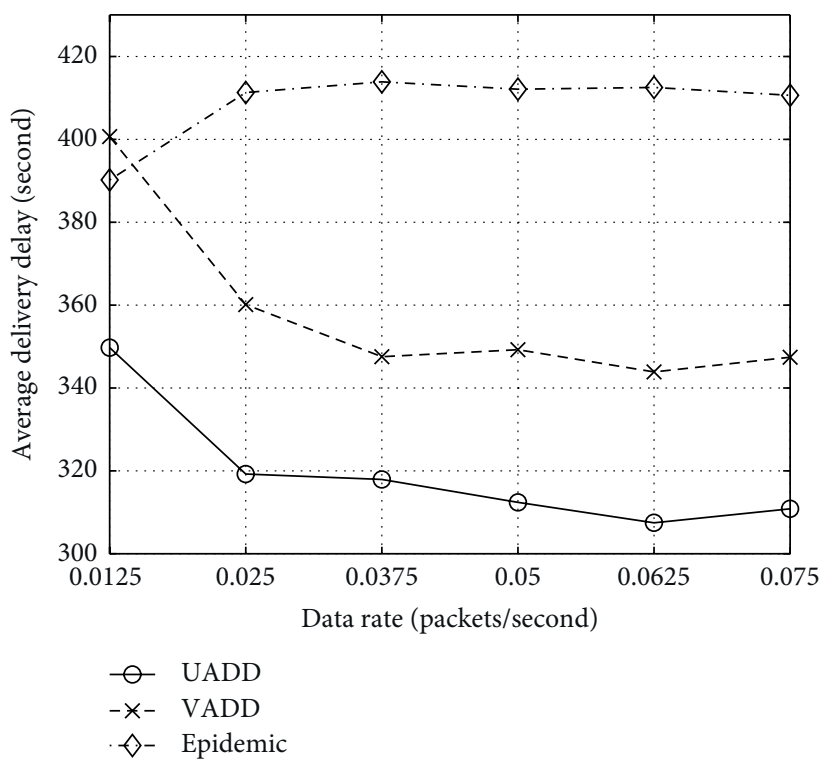

Figure 8: Average packet delivery delay. Buffers of vehicles are set to 50 packets.

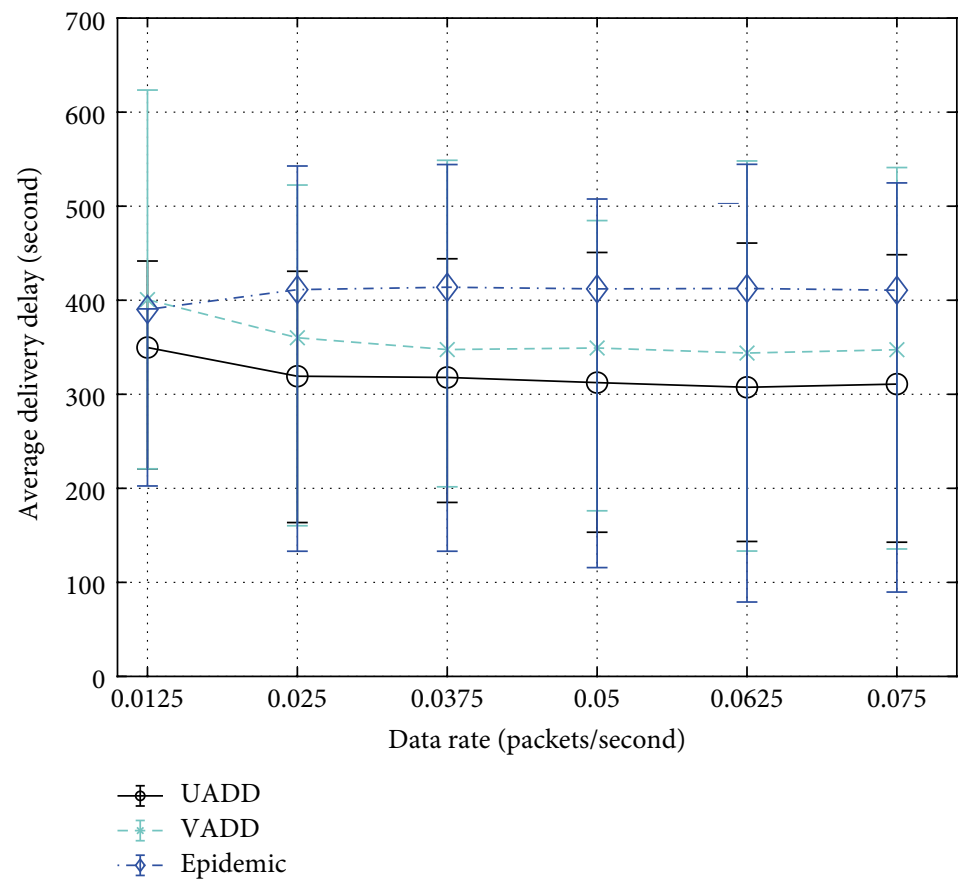

FIgURE 9: Average delay with CI.

delay of Epidemic can be seen with the data rate increasing from $0.0125 \mathrm{packet} / \mathrm{second}$ to $0.025 \mathrm{packet} / \mathrm{second}$, because buffers are less saturated when the data rate is 0.0125 packet/ second. In addition, the proposed UADD method can achieve lower average packet delivery delay. This is because UAVs can provide packet forwarding opportunities with lower expected delivery delay according to (4). The decrease in average packet delivery delay of both UADD and VADD when data rate is low is because when the number of packets in the network is small, many packets may not be able to find the optimal route to the destination. Besides, the CI of the packet delay is shown in Figure 9. Note that the studies scenario in the paper is the delay-tolerant case, which indicates the delay distribution is with long-tail. This explains why the CI is large in the figure.

4.3. The Data Traffic Overhead. Figure 10 shows the data traffic overhead of different routing protocols. The average packets generated per second in the network is used as the metric to evaluate the data traffic overhead of corresponding protocol. In Figure 10(a), the data traffic overhead with respect to the data rate is depicted. It can be seen that for all protocol, UADD, VADD, and Epidemic, the data traffic increases with 


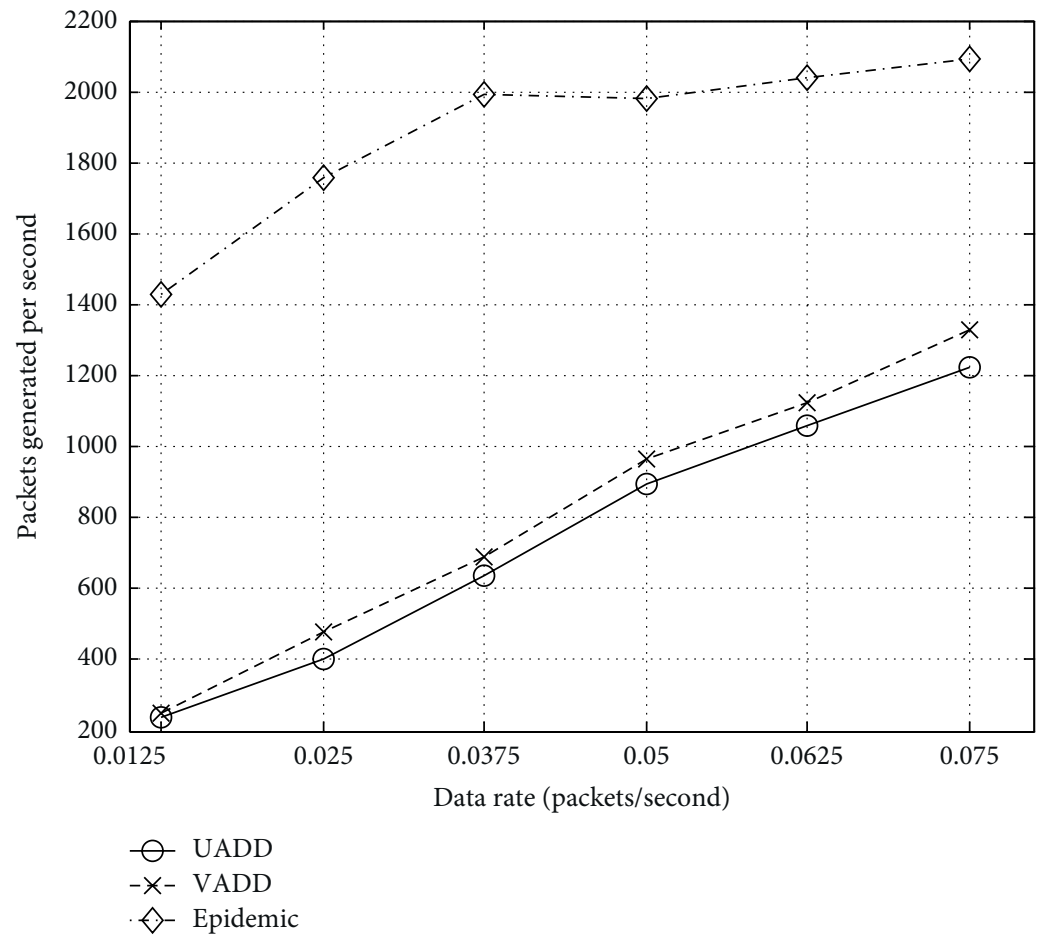

(a)

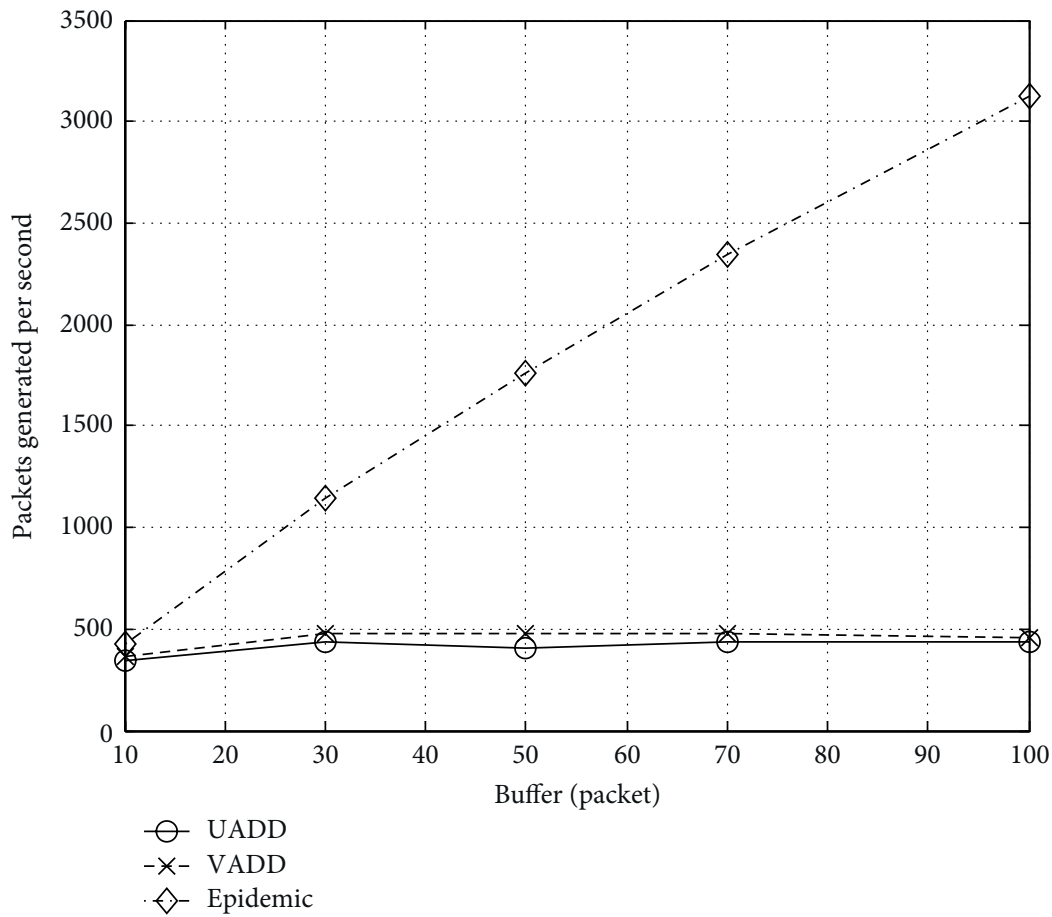

(b)

Figure 10: Data traffic overhead of routing protocols. (a) Data traffic overhead vs. data rate. (b) Data traffic overhead vs. packet buffer.

the data rate, which is straightforward since with higher data rate, more packets are generated by each vehicle. However, the data traffic overhead using Epidemic protocol is much higher than that of UADD and VADD, because in Epidemic protocol, each packet is copied and transmitted to achieve better delivery performance, while in UADD and VADD each packet has only one copy in the network. The slightly difference between UADD and VADD is because only the packets carried by ground vehicles are used to calculate the data traffic overhead. In UADD, some packets are carried and forwarded by UAVs. Figure 10(b) shows the data traffic overhead versus the maximum packet buffer size of vehicles. We can see from the figure that the data traffic overhead remains the same with the increase of buffer size for UADD and VADD. This is 
because due to the one-copy mechanism of both protocols, the vehicle packet buffers do not use up. However, for Epidemic, the buffers are full by storing the copies of packets, and thus the data traffic overhead increases linearly with the buffer size. From the results, Epidemic protocol generates much higher data traffic than UADD and VADD, which will lead to more packet collision, drops, and consumes more power.

\section{Conclusion}

In this paper, we have designed a UAV-aided data delivery scheme for smart transportation networks in urban scenarios. Acting as relays or packet carriers, UAVs can offer more opportunities to deliver the packets. The packet forwarding rules have been derived based on delivery delay and probability. Simulations have been performed to validate the performance of the proposed UADD scheme, in comparison with the existing routing protocols. However, we do not derive the theoretical analysis on the performance, since the forwarding probability of data packet depends on the vehicle mobility and the UAV trajectories, which is difficult to calculate in theory. For future work, scheduling routes for UAVs will be considered to optimize the delivery performance. We also plan to utilize AI learning and prediction in optimizing our method. In addition, more practical scenarios can be considered, for example, the routes of UAVs can intersect with the intersection, and packet forwarding can be more complicated.

\section{Data Availability}

The data reported in our submission were generated during the study.

\section{Conflicts of Interest}

The authors declare that they have no conflicts of interest.

\section{Acknowledgments}

This work was supported by the National Natural Science Foundation of China (61701170), Henan International Science and Technology Cooperation Program (182102410050), and Key Project of Science and Technology Research of the Education Department of Henan Province (17A413001, 17A120007).

\section{References}

[1] L. W. Lan and C. Chang, "Inhomogeneous cellular automata modelling for mixed traffic with cars and motorcycles," Journal of Advanced Transportation, vol. 39, no. 3, pp. 323-349, 2010.

[2] N. Lu, N. Cheng, N. Zhang, X. Shen, and J. W. Mark, "Connected vehicles: solutions and challenges," IEEE Internet of Things Journal, vol. 1, no. 4, pp. 289-299, 2014.
[3] P. R. Pereira, A. Casaca, J. J. P. C. Rodrigues, V. N. G. J. Soares, J. Triay, and C. Cervello-Pastor, "From delay-tolerant networks to vehicular delay-tolerant networks," IEEE Communications Surveys \& Tutorials, vol. 14, no. 4, pp. 1166-1182, 2012.

[4] J. N. G. Isento, J. J. P. C. Rodrigues, J. A. F. F. Dias, M. C. G. Paula, and A. Vinel, "Vehicular delay-tolerant networks? a novel solution for vehicular communications," IEEE Intelligent Transportation Systems Magazine, vol. 5, no. 4, pp. 10-19, 2013.

[5] N. Benamar, K. D. Singh, M. Benamar, D. El Ouadghiri, and J.-M. Bonnin, "Routing protocols in vehicular delay tolerant networks: a comprehensive survey," Computer Communications, vol. 48, no. 8, pp. 141-158, 2014.

[6] J. Zhao and G. Cao, "VADD: vehicle-assisted data delivery in vehicular ad hoc networks" IEEE Transactions on Vehicular Technology, vol. 57, no. 3, pp. 1910-1922, 2006.

[7] Y. Li, Y. Jiang, D. Jin, S. Li, L. Zeng, and D. Wu, "Energy-efficient optimal opportunistic forwarding for delay-tolerant networks," IEEE Transactions on Vehicular Technology, vol. 59, no. 9, pp. 4500-4512, 2010.

[8] N. Cheng, N. Lu, N. Zhang, T. T. Yang, X. Shen, and J. W. Mark, "Vehicle-assisted device-to-device data delivery for smart grid," IEEE Transactions on Vehicular Technology, vol. 65, no. 4, pp. 2325-2340, 2016.

[9] N. Cheng, H. Zhou, L. Lei et al., "Performance analysis of vehicular device-to-device underlay communication," IEEE Transactions on Vehicular Technology, vol. 66, no. 6, pp. 54095421, 2017.

[10] O. Esrafilian and D. Gesbert, "3D city map reconstruction from UAV-based radio measurements, in GLOBECOM 2017-2017," in IEEE Global Communications Conference, pp. 1-6, 2017.

[11] Y. Yang, Z. Zheng, K. Bian, Y. Jiang, L. Song, and Z. Han, "Arms: a fine-grained 3D AQI realtime monitoring system by UAV, in GLOBECOM 2017-2017," IEEE Global Communications Conference, pp. 1-6, 2017.

[12] A. Souppouris, "Renault concept car launches drone to check for gridlock ahead. renault-kwiddrone-car-concept," 2014, http:// www.theverge.com/2014/2/7/5389114/.

[13] Y. Zhou, N. Cheng, N. Lu, and X. Shen, "Multi-UAV-aided networks: aerial-ground cooperative vehicular networking architecture," IEEE Vehicular Technology Magazine, vol. 10, no. 4, pp. 36-44, 2015.

[14] J. Ueyama, H. Freitas, B. S. Faical et al., "Exploiting the use of unmanned aerial vehicles to provide resilience in wireless sensor networks," IEEE Communications Magazine, vol. 52, no. 12, pp. 81-87, 2014.

[15] J. Wang, J. Lv, C. Wang, and Z. Zhang, "Dynamic route choice prediction model based on connected vehicle guidance characteristics," Journal of Advanced Transportation, vol. 2017, no. 1, pp. 1-8, 2017.

[16] M. Tortonesi, C. Stefanelli, E. Benvegnu, K. Ford, N. Suri, and M. Linderman, "Multiple-UAV coordination and communications in tactical edge networks," IEEE Communications Magazine, vol. 50, no. 10, pp. 48-55, 2012.

[17] Z. Han, A. L. Swindlehurst, and K. J. R. Liu, "Optimization of MANET connectivity via smart deployment/movement of unmanned air vehicles," IEEE Transactions on Vehicular Technology, vol. 58, no. 7, pp. 3533-3546, 2009.

[18] N. Goddemeier, D. Kai, and C. Wietfeld, "Role-based connectivity management with realistic air-to-ground channels for cooperative UAVs," IEEE Journal on Selected Areas in Communications, vol. 30, no. 5, pp. 951-963, 2012. 
[19] H. Menouar, I. Guvenc, K. Akkaya, A. S. Uluagac, A. Kadri, and A. Tuncer, "UAV-enabled intelligent transportation systems for the smart city: applications and challenges," IEEE Communications Magazine, vol. 55, no. 3, pp. 22-28, 2017.

[20] T. V. Woensel and N. Vandaele, "Modeling traffic flows with queueing models: a review," Asia-Pacific Journal of Operational Research, vol. 24, no. 4, pp. 435-461, 2007.

[21] J. Härri, F. Filali, C. Bonnet, and M. Fiore, "VanetMobiSim: generating realistic mobility patterns for VANETs," in Proceedings of the 3rd International Workshop on Vehicular Ad Hoc Networks, VANET’06, pp. 96-97, USA, 2006. 


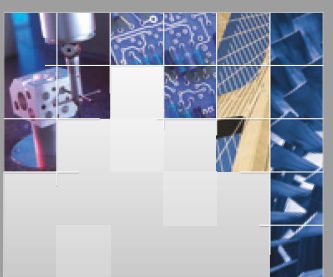

\section{Enfincering}
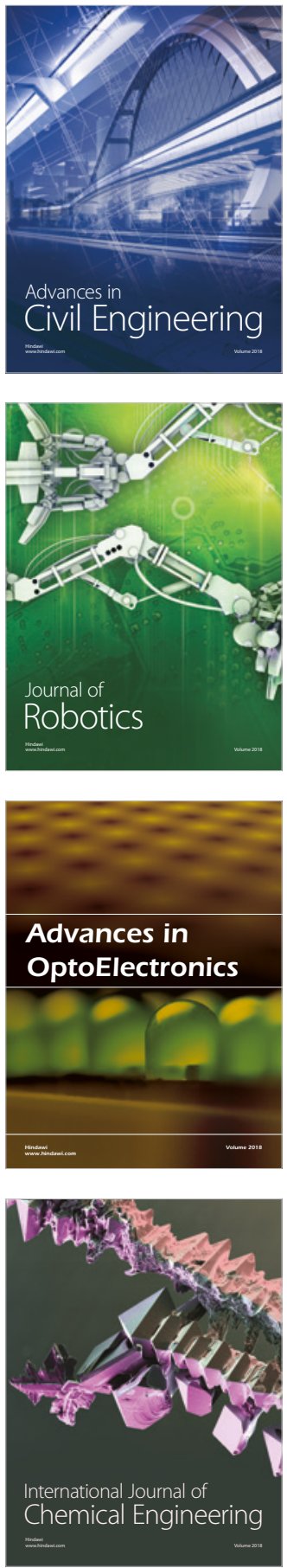

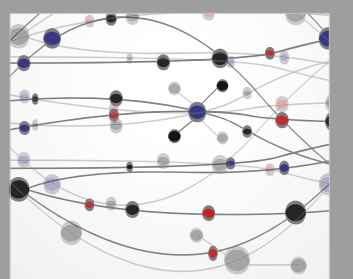

\section{Rotating \\ Machinery}

The Scientific World Journal

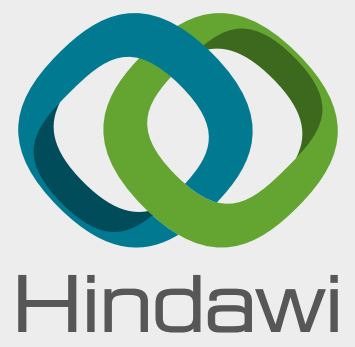

Submit your manuscripts at

www.hindawi.com
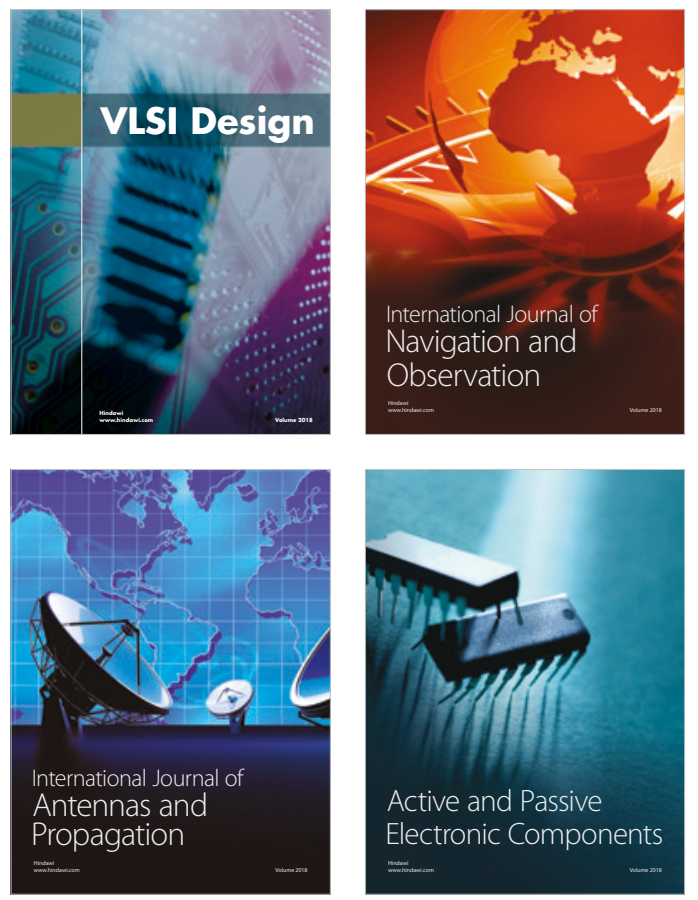
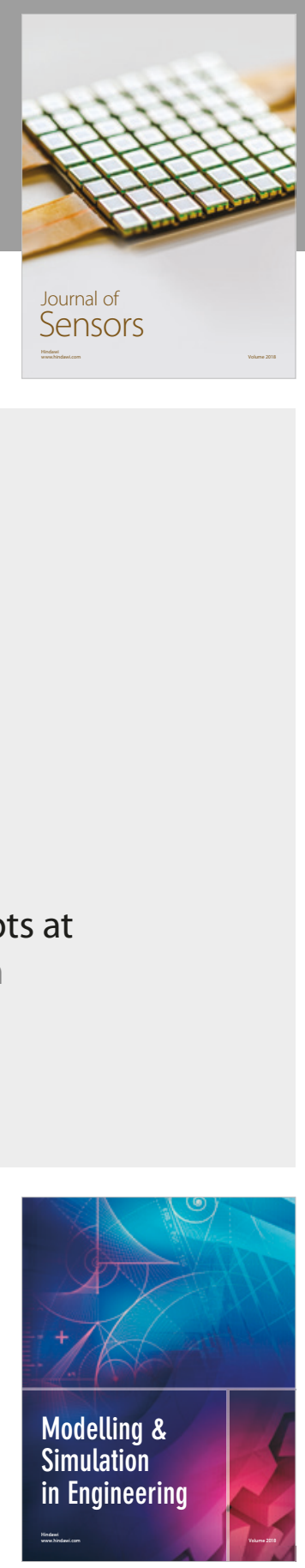

\section{Advances \\ Multimedia}
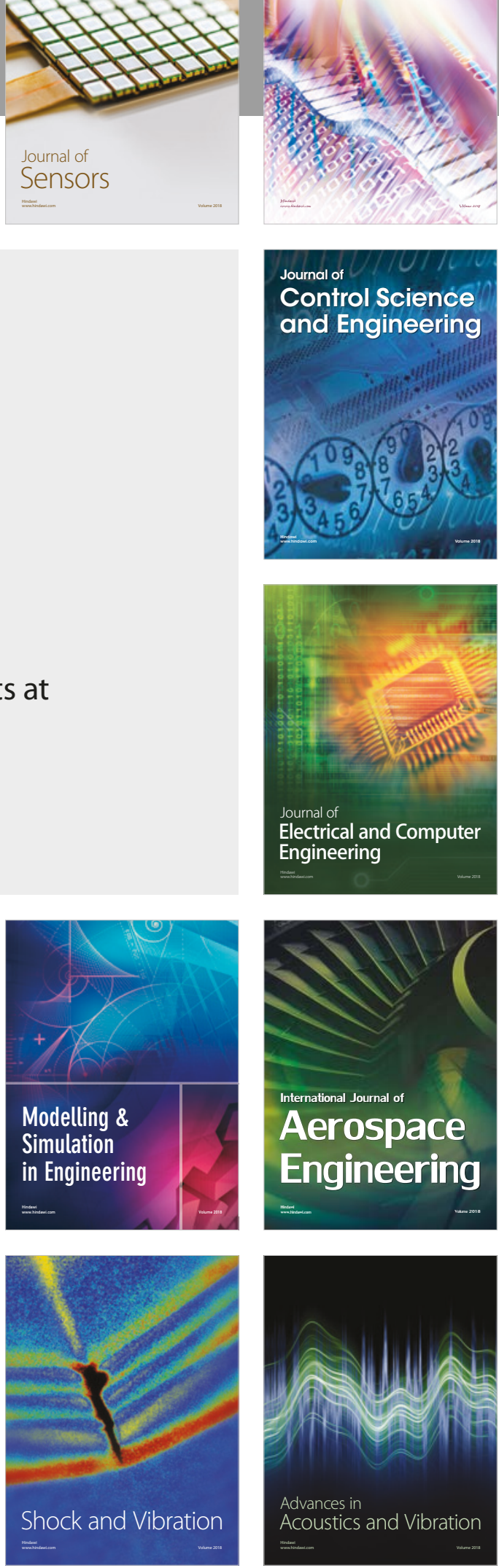